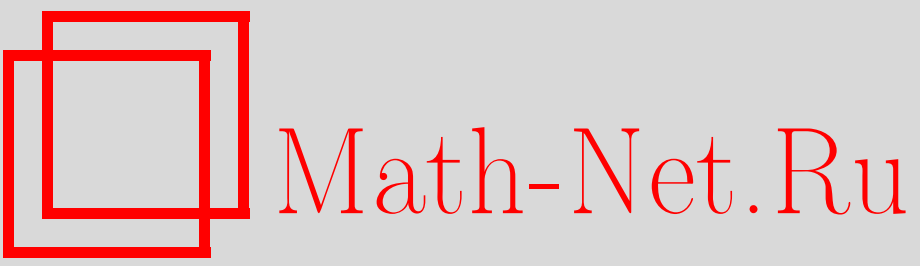

В. Д. Гордевский, Двухпотоковое распределение с винтовыми модами, ТМФ, 2001, том 126, номер 2, 283-300

DOI: https://doi.org/10.4213/tmf431

Использование Общероссийского математического портала Math-Net.Ru подразумевает, что вы прочитали и согласны с пользовательским соглашением

http://www.mathnet.ru/rus/agreement

Параметры загрузки:

IP : 3.81 .55 .215

26 апреля 2023 г., 16:41:37 
ТЕОРЕТИЧЕСКАЯ

И МАТЕМАТИЧЕСКАЯ

ФИЗИКА

Том 126, № 2

февраль, 2001

(c) 2001 г.

\author{
В. Д. Гордевский*
}

\title{
ДВУХПОТОКОВОЕ РАСПРЕДЕЛЕНИЕ С ВИНТОВЫМИ МОДАМИ
}

Для модели твердых сфер построено бимодальное распределение, имеющее вид линейной комбинации стационарных неоднородных максвеллианов. Оно описывает взаимодействие двух потоков газа, совершающих вращательное движение вокруг неподвижных осей и поступательное движение вдоль них. Найдены предельные случаи, в которых это распределение минимизирует равномерно-интегральную невязку между частями уравнения Больцмана.

\section{1. ВВЕДЕНИЕ}

При описании эволюции разреженного газа из твердых сфер используется кинетическое уравнение Больцмана [1], [2]

$$
\begin{gathered}
D(f)=Q(f, f), \\
D(f)=\frac{\partial f}{\partial t}+v \frac{\partial f}{\partial x} \\
Q(f, f)=\frac{d^{2}}{2} \int_{\mathbb{R}^{3}} d v_{1} \int_{\Sigma} d \alpha\left|\left(v_{1}-v, \alpha\right)\right|\left[f\left(t, v_{1}^{\prime}, x\right) f\left(t, v^{\prime}, x\right)-f\left(t, v_{1}, x\right) f(t, v, x)\right],
\end{gathered}
$$

где $f$ - искомая функция распределения молекул, $\partial f / \partial x$ - ее пространственный градиент, $t \in \mathbb{R}^{1}$ - время, $x=\left(x^{1}, x^{2}, x^{3}\right) \in \mathbb{R}^{3}$ и $v=\left(v^{1}, v^{2}, v^{3}\right) \in \mathbb{R}^{3}$ - координата и скорость молекулы, $d$ - ее диаметр, $\alpha \in \Sigma, \Sigma$ - единичная сфера в $\mathbb{R}^{3}$. Скорости молекул после столкновения задаются формулами

$$
v^{\prime}=v-\alpha\left(v-v_{1}, \alpha\right), \quad v_{1}^{\prime}=v_{1}+\alpha\left(v-v_{1}, \alpha\right) .
$$

Известными точными решениями уравнения (1)-(4) являются глобальные и локальные максвеллианы [1], [2]. Другие точные решения получены только для случая максвелловских молекул и некоторых его обобщений [3]-[14].

В работах [15]-[18] осуществлено приближенное описание взаимодействия двух потоков газа из твердых сфер, соответствуюших глобальным (т.е. не зависящим ни от $t$, ни от

* Харьковский национальный университет, Харьков, Украина 
$x)$ максвеллианам. При этом в качестве невязки между частями уравнения Больцмана использовалась смешанная (равномерная по $t, x$ и интегральная по $v$ ) норма разности $D(f)-Q(f, f)$. Несколько иной подход с использованием чисто интегральной метрики предложен в [19].

Попытки переноса результатов этих работ на случай локальных максвеллианов наиболее общего вида пока не увенчались успехом ввиду возникающих при этом значительных технических трудностей.

Целью данной работы является изучение поведения бимодального распределения, в которое входят локальные максвеллианы частного вида, описываюшие винтообразные стационарные равновесные состояния газа (для краткости называемые в дальнейшем просто винты). Каждый такой максвеллиан задается формулой [1]

$$
M(v, x)=\rho_{0} e^{\beta \omega^{2} r^{2}}\left(\frac{\beta}{\pi}\right)^{\frac{3}{2}} e^{-\beta(v-\bar{v}-[\omega \times x])^{2}} .
$$

С физической точки зрения распределение (5) описывает врашение газа как целого (наподобие твердого тела) с угловой скоростью $\omega \in \mathbb{R}^{3}$ вокруг оси, проходящей через точку

$$
x_{0}=\frac{[\omega \times \bar{v}]}{\omega^{2}},
$$

$x_{0} \in \mathbb{R}^{3}$, причем $\beta=1 / 2 T$ - обратная температура газа,

$$
r^{2}=\frac{1}{\omega^{2}}\left[\omega \times\left(x-x_{0}\right)\right]^{2}
$$

- квадрат расстояния до оси врашения

$$
\rho=\rho_{0} e^{\beta \omega^{2} r^{2}}
$$

- плотность газа ( $\rho_{0}$ - плотность на оси врашения, т.е. при $\left.r=0\right), \bar{v} \in \mathbb{R}^{3}$ - линейная массовая скорость в точках $x$, для которых $x \| \omega$, а $\bar{v}+[\omega \times x]$ - массовая скорость в произвольной точке $x$. Легко видеть, что формула (5) помимо врашательного задает и поступательное движение вдоль оси врашения с линейной скоростью

$$
\frac{(\omega, \bar{v})}{\omega^{2}} \omega,
$$

т.е. действительно описывает винтообразное движение газа в целом, причем это распределение стационарно (не зависит от $t$ ), но неоднородно.

Постановка задачи такова. Будем рассматривать неоднородную, нестационарную линейную комбинацию двух максвеллианов вида (5), т.е. распределение

$$
\begin{gathered}
f=\varphi_{1} M_{1}+\varphi_{2} M_{2}=\sum_{i=1}^{2} \varphi_{i}(t, x) M_{i}(v, x), \\
M_{i}(v, x)=\rho_{i} e^{\beta_{i} \omega_{i}^{2} r_{i}^{2}}\left(\frac{\beta_{i}}{\pi}\right)^{\frac{3}{2}} e^{-\beta_{i}\left(v-\tilde{v}_{i}\right)^{2}} \\
\tilde{v}_{i}=\tilde{v}_{i}(x)=\bar{v}_{i}+\left[\omega_{i} \times x\right], \quad i=1,2 .
\end{gathered}
$$


Коэффициентные функции $\varphi_{i}, i=1,2$, предполагаются неотрицательными и принадлежашими $C^{1}\left(\mathbb{R}^{4}\right)$. Требуется найти такие функции $\varphi_{i}$ и такое поведение всех имеюшихся параметров, чтобы “смешанная" невязка

$$
\Delta=\sup _{(t, x) \in \mathbb{R}^{4}} \int_{\mathbb{R}^{3}}|D(f)-Q(f, f)| d v
$$

стремилась при этом к нулю.

В разделе 2 получены асимптотические выражения некоторых оценок сверху для невязки $\Delta$ при больших значениях $\beta_{1}, \beta_{2}$ и различных предположениях о поведении векторов $\omega_{i}$ и $\bar{v}_{i}, i=1,2$. В разделе 3 найдены некоторые условия, достаточные для стремления величины $\Delta$ к нулю. Раздел 4 посвяшен обсуждению полученных результатов.

\section{2. АСИМПТОТИКА ВЕЛИЧИНЫ $\Delta$ ПРИ БОЛЬШИХ $\beta_{1}, \beta_{2}$}

В данном разделе изучается поведение невязки $\Delta$ при возрастании параметров $\beta_{i}$, $i=1,2$. С физической точки зрения это соответствует стремлению максвеллианов, входящих в распределение (9)-(11), к $\delta$-функциям, т.е. локализации скоростей частиц вблизи соответствующих массовых скоростей в каждой точке пространства. Формально поиск такой асимптотики сводится к вычислению предела величины $\Delta$ (либо ее оценки сверху) при $\beta_{i} \rightarrow+\infty, i=1,2$. Для этого необходимо несколько преобразовать правую часть (12). Прежде всего вычислим и оценим интеграл по переменной $v$, подставляя в (1)-(3) распределение (9)-(11) и учитывая, что

$$
D\left(M_{i}\right)=Q\left(M_{i}, M_{i}\right)=0, \quad i=1,2 .
$$

Имеем

$$
\begin{gathered}
D(f)=D\left(\varphi_{1}\right) M_{1}+D\left(\varphi_{2}\right) M_{2}=\sum_{i=1}^{2} D\left(\varphi_{i}\right) e^{\beta_{i} \omega_{i}^{2} r_{i}^{2}} \widetilde{M}_{i} \\
Q(f, f)=\varphi_{1} \varphi_{2} e^{\beta_{1} \omega_{1}^{2} r_{1}^{2}} e^{\beta_{2} \omega_{2}^{2} r_{2}^{2}}\left[Q\left(\widetilde{M}_{1}, \widetilde{M}_{2}\right)+Q\left(\widetilde{M}_{2}, \widetilde{M}_{1}\right)\right]
\end{gathered}
$$

где введено обозначение

$$
\widetilde{M}_{i}=\widetilde{M}_{i}(v, x)=\rho_{i}\left(\frac{\beta_{i}}{\pi}\right)^{\frac{3}{2}} e^{-\beta i\left(v-\tilde{v}_{i}\right)^{2}}
$$

Тогда

$$
\begin{aligned}
\int_{\mathbb{R}^{3}}|D(f)-Q(f, f)| d v= & \int_{\mathbb{R}^{3}} \mid \sum_{i=1}^{2} D\left(\varphi_{i}\right) e^{\beta_{i} \omega_{i}^{2} r_{i}^{2}} \widetilde{M}_{i}- \\
& -\varphi_{1} \varphi_{2} e^{\beta_{1} \omega_{1}^{2} r_{1}^{2}} e^{\beta_{2} \omega_{2}^{2} r_{2}^{2}} \sum_{\substack{i, j=1 \\
i \neq j}}^{2} Q\left(\widetilde{M}_{i}, \widetilde{M}_{j}\right) \mid d v .
\end{aligned}
$$


Произведя, как обычно (см., например, [1], [15]), разбиение интеграла столкновений $Q$ на "прибыточную" и “затратную" части $G$ и $L$ (что соответствует раскрытию квадратной скобки в (3)) и учитывая, что $G \geqslant 0$, для выражения (17) получим следуюшую оценку сверху:

$$
\begin{aligned}
\int_{\mathbb{R}^{3}}|D(f)-Q(f, f)| d v \leqslant & \sum_{\substack{i, j=1 \\
i \neq j}}^{2}\left[\int_{\mathbb{R}^{3}}\left|D\left(\varphi_{i}\right) e^{\beta_{i} \omega_{i}^{2} r_{i}^{2}}+\varphi_{1} \varphi_{2} e^{\beta_{1} \omega_{1}^{2} r_{1}^{2}} e^{\beta_{2} \omega_{2}^{2} r_{2}^{2}} L\left(\widetilde{M}_{j}\right)\right| \widetilde{M}_{i} d v+\right. \\
& \left.+\varphi_{1} \varphi_{2} e^{\beta_{1} \omega_{1}^{2} r_{1}^{2}} e^{\beta_{2} \omega_{2}^{2} r_{2}^{2}} \int_{\mathbb{R}^{3}} G\left(\widetilde{M}_{i}, \widetilde{M}_{j}\right) d v\right]
\end{aligned}
$$

Поскольку, как известно [1], справедливо равенство

$$
\int_{\mathbb{R}^{3}} G\left(\widetilde{M}_{i}, \widetilde{M}_{j}\right) d v=\int_{\mathbb{R}^{3}} L\left(\widetilde{M}_{j}\right) \widetilde{M}_{i} d v, \quad i=1,2, \quad i \neq j,
$$

то в формуле (18) последний интеграл заменим на правую часть (19). Далее, так как (см. [15])

$$
L\left(\widetilde{M}_{j}\right)=\rho_{j} \frac{d^{2}}{\sqrt{\pi}} \int_{\mathbb{R}^{3}} d w\left|v-\tilde{v}_{j}-\frac{w}{\sqrt{\beta_{j}}}\right| e^{-w^{2}},
$$

можно первый интеграл из $i$-го слагаемого в $(18)$ с учетом (2) и (16) представить в виде

$$
\begin{aligned}
\int_{\mathbb{R}^{3}} \mid & \left(\frac{\partial \varphi_{i}}{\partial t}+v \frac{\partial \varphi_{i}}{\partial x}\right) e^{\beta_{i} \omega_{i}^{2} r_{i}^{2}}+\varphi_{1} \varphi_{2} e^{\beta_{1} \omega_{1}^{2} r_{1}^{2}} e^{\beta_{2} \omega_{2}^{2} r_{2}^{2}} \rho_{j} \frac{d^{2}}{\sqrt{\pi}} \times \\
& \times \int_{\mathbb{R}^{3}} d w e^{-w^{2}}\left|v-\tilde{v}_{j}-\frac{w}{\sqrt{\beta_{j}}}\right| \mid \rho_{i}\left(\frac{\beta_{i}}{\pi}\right)^{\frac{3}{2}} e^{-\beta_{i}\left(v-\tilde{v}_{i}\right)^{2}} d v .
\end{aligned}
$$

После замены

$$
u=\sqrt{\beta_{i}}\left(v-\tilde{v}_{i}\right)
$$

и подстановки выражений (11) получим

$$
\begin{aligned}
& \int_{\mathbb{R}^{3}} \mid\left(\frac{\partial \varphi_{i}}{\partial t}+\left(\frac{u}{\sqrt{\beta_{i}}}+\bar{v}_{i}+\left[\omega_{i} \times x\right]\right) \frac{\partial \varphi_{i}}{\partial x}\right) e^{\beta_{i} \omega_{i}^{2} r_{i}^{2}}+\varphi_{1} \varphi_{2} e^{\beta_{1} \omega_{1}^{2} r_{1}^{2}} e^{\beta_{2} \omega_{2}^{2} r_{2}^{2} \times} \\
& \quad \times \rho_{j} \frac{d^{2}}{\sqrt{\pi}} \int_{\mathbb{R}^{3}} d w e^{-w^{2}}\left|\frac{u}{\sqrt{\beta_{i}}}-\bar{v}_{j}-\left[\omega_{j} \times x\right]+\bar{v}_{i}+\left[\omega_{i} \times x\right]-\frac{w}{\sqrt{\beta_{j}}}\right| \mid \rho_{i}\left(\frac{1}{\pi}\right)^{\frac{3}{2}} e^{-u^{2}} d u
\end{aligned}
$$

(второй интеграл в (18) преобразуется совершенно аналогично).

Из (18) и (23) видно, что для корректной определенности невязки (12) на коэффициентные функции $\varphi_{i}$ следует наложить новые условия типа финитности или быстрого убывания по пространственной переменной $x$. Поэтому будем искать их в виде

$$
\varphi_{i}(t, x)=\psi_{i}(t, x) e^{-\beta_{i} \omega_{i}^{2} r_{i}^{2}}, \quad i=1,2,
$$


где функции $\psi_{i}$ гладкие и неотрицательные (предполагается, что они уже не зависят от $\left.\beta_{i}\right)$. Тогда оценка $(18)$ с учетом $(23),(24)$ и $(7)$ приобретает вид

$$
\begin{aligned}
& \int_{\mathbb{R}^{3}}|D(f)-Q(f, f)| d v \leqslant \sum_{\substack{i, j=1 \\
i \neq j}}^{2}\left[\int_{\mathbb{R}^{3}} \mid \frac{\partial \psi_{i}}{\partial t}+\left(\frac{u}{\sqrt{\beta_{i}}}+\bar{v}_{i}+\left[\omega_{i} \times x\right]\right) \times\right. \\
& \quad \times\left(\frac{\partial \psi_{i}}{\partial x}-2 \beta_{i} \psi_{i}\left[\left[\omega_{i} \times\left(x-x_{0 i}\right)\right] \times \omega_{i}\right]\right)+ \\
& \quad+\psi_{1} \psi_{2} \rho_{j} \frac{d^{2}}{\sqrt{\pi}} \int_{\mathbb{R}^{3}} d w e^{-w^{2}}\left|\frac{u}{\sqrt{\beta_{i}}}+\left(\bar{v}_{i}-\bar{v}_{j}\right)+\left[\left(\omega_{i}-\omega_{j}\right) \times x\right]-\frac{w}{\sqrt{\beta_{j}}}\right| \mid \frac{\rho_{i}}{\pi^{\frac{3}{2}}} e^{-u^{2}} d u+ \\
& \left.\quad+\psi_{1} \psi_{2} \frac{\rho_{1} \rho_{2} d^{2}}{\pi^{2}} \int_{\mathbb{R}^{3}} e^{-u^{2}} d u \int_{\mathbb{R}^{3}} d w e^{-w^{2}}\left|\frac{u}{\sqrt{\beta_{i}}}+\left(\bar{v}_{i}-\bar{v}_{j}\right)+\left[\left(\omega_{i}-\omega_{j}\right) \times x\right]-\frac{w}{\sqrt{\beta_{j}}}\right|\right] .
\end{aligned}
$$

Исходя из (25) получим некоторые условия, налагаемые на функции $\psi_{i}, i=1,2$, и на входяшие в распределение (9)-(11) параметры, достаточные для корректной определенности невязки $\Delta$, а также для сушествования предела при $\beta_{i} \rightarrow+\infty, i=1,2$.

Прежде чем формулировать и доказывать основные результаты, упростим выражение (25). Во-первых, положим

$$
\begin{gathered}
A_{i}(u, t, x)=\psi_{1} \psi_{2} \rho_{j} \frac{d^{2}}{\sqrt{\pi}} \int_{\mathbb{R}^{3}} d w e^{-w^{2}}\left|\frac{u}{\sqrt{\beta_{i}}}+\left(\bar{v}_{i}-\bar{v}_{j}\right)+\left[\left(\omega_{i}-\omega_{j}\right) \times x\right]-\frac{w}{\sqrt{\beta_{j}}}\right|, \\
B_{i}(u, t, x)=\left(\frac{u}{\sqrt{\beta_{i}}}+\bar{v}_{i}+\left[\omega_{i} \times x\right]\right)\left(\frac{\partial \psi_{i}}{\partial x}+2 \beta_{i} \psi_{i}\left[\omega_{i} \times\left[\omega_{i} \times\left(x-x_{0 i}\right)\right]\right]\right),
\end{gathered}
$$

где $i, j=1,2, i \neq j$. Тогда (25) можно переписать в виде

$$
\begin{aligned}
\int_{\mathbb{R}^{3}}|D(f)-Q(f, f)| d v \leqslant & \sum_{i=1}^{2} \int_{\mathbb{R}^{3}}\left[\left|\frac{\partial \psi_{i}}{\partial t}+B_{i}(u, t, x)+A_{i}(u, t, x)\right|+\right. \\
& \left.+A_{i}(u, t, x)\right] \frac{\rho_{i}}{\pi^{\frac{3}{2}}} e^{-u^{2}} d u
\end{aligned}
$$

Во-вторых, преобразуем выражение для $B_{i}(u, t, x)$, опираясь на известные формулы векторной алгебры

$$
\begin{aligned}
{[a \times[b \times c]] } & =b(a, c)-c(a, b), \\
{[a \times b] \cdot[a \times c] } & =a^{2}(b, c)-(a, b)(a, c),
\end{aligned}
$$


где $a, b, c \in \mathbb{R}^{3}$ - произвольные векторы. Тогда с учетом (6) имеем

$$
\begin{aligned}
B_{i}(u, t, x)= & \left.\frac{u}{\sqrt{\beta_{i}}}+\bar{v}_{i}+\left[\omega_{i} \times x\right]\right) \times \\
& \times\left\{\frac{\partial \psi_{i}}{\partial x}+2 \beta_{i} \psi_{i}\left(\omega_{i}\left(\omega_{i}, x-x_{0 i}\right)-\omega_{i}^{2}\left(x-\frac{\left[\omega_{i} \times \bar{v}_{i}\right]}{\omega_{i}^{2}}\right)\right)\right\}= \\
= & \left.\frac{u}{\sqrt{\beta_{i}}}+\bar{v}_{i}+\left[\omega_{i} \times x\right]\right) \times \\
& \times\left\{\frac{\partial \psi_{i}}{\partial x}+2 \beta_{i} \psi_{i}\left(\omega_{i}\left(\omega_{i}, x\right)-x \omega_{i}^{2}+\left[\omega_{i} \times \bar{v}_{i}\right]\right)\right\}= \\
= & \frac{\partial \psi_{i}}{\partial x}\left(\frac{u}{\sqrt{\beta_{i}}}+\bar{v}_{i}+\left[\omega_{i} \times x\right]\right)+ \\
& +2 \psi_{i} \sqrt{\beta_{i}}\left\{\left(\omega_{i}, u\right)\left(\omega_{i}, x\right)-\omega_{i}^{2}(x, u)+\left(u,\left[\omega_{i} \times \bar{v}_{i}\right]\right)\right\}= \\
= & \frac{\partial \psi_{i}}{\partial x}\left(\frac{u}{\sqrt{\beta_{i}}}+\bar{v}_{i}+\left[\omega_{i} \times x\right]\right)+ \\
& +2 \psi_{i} \sqrt{\beta_{i}}\left\{-\left[\omega_{i} \times u\right] \cdot\left[\omega_{i} \times x\right]+\left(u,\left[\omega_{i} \times \bar{v}_{i}\right]\right)\right\} .
\end{aligned}
$$

TEOPEMA 1. Пyсmь

$$
\omega_{i}=\frac{\omega_{0 i} s_{i}}{\beta_{i}}, \quad i=1,2,
$$

где $s_{i}>0$ - любие константы, $\omega_{0 i}$ - произвольные фиксированные векторы (остальные параметры также произвольны и фиксированы). Тогда, если величины

$$
\psi_{i}, \quad \frac{\partial \psi_{i}}{\partial t}, \quad\left|\frac{\partial \psi_{i}}{\partial x}\right|, \quad\left|\left[\omega_{0 i} \times x\right]\right| \psi_{i}, \quad\left(\left[\omega_{0 i} \times x\right], \frac{\partial \psi_{i}}{\partial x}\right), \quad i=1,2,
$$

ограничены по $t, x$ на $\mathbb{R}^{4}$, то существует $\Delta$, определенное в соответствии с (12), и maкое $\Delta^{\prime}$, чmo

$$
\Delta \leqslant \Delta^{\prime}
$$

причем

$$
\begin{gathered}
\lim _{\substack{\beta_{i} \rightarrow+\infty \\
i=1,2}} \Delta^{\prime}=\sum_{\substack{i, j=1 \\
i \neq j}}^{2} \rho_{i} \sup _{(t, x) \in \mathbb{R}^{4}}\left|\frac{\partial \psi_{i}}{\partial t}+\bar{v}_{i} \frac{\partial \psi_{i}}{\partial x}+\rho_{j} \pi d^{2} \psi_{1} \psi_{2}\right| \bar{v}_{i}-\bar{v}_{j}||+ \\
+2 \pi d^{2} \rho_{1} \rho_{2}\left|\bar{v}_{1}-\bar{v}_{2}\right| \sup _{(t, x) \in \mathbb{R}^{4}}\left(\psi_{1} \psi_{2}\right) .
\end{gathered}
$$

Для доказательства теоремы 1 нам понадобится следующая лемма, даюшая достаточное условие непрерывности супремума специального вида функции многих переменных, взятого по части переменных.

ЛЕмма 1. Пусть функиии $f(y), L_{k}(z, y), k=1, \ldots, m$, определень при $y \in Y \subseteq$ $\mathbb{R}^{p}, \quad z \in Z \subseteq \mathbb{R}^{q}$ и удовлетворяют условиям:

1) $f(y)$ ограничена на $Y$; 
2) $\forall z \in Z, \forall k, k \in\{1, \ldots, m\}, L_{k}(z, y)$ ограничена на $Y$;

3) $\forall k, k=1, \ldots, m, L_{k}(z, y)$ непрерывна по z на $Z$ равномерно относительно у на $Y$, m.e.

$\forall z_{0} \in Z, \forall \varepsilon>0 \exists \delta>0: \forall y \in Y, \forall z \in Z,\left|z-z_{0}\right|<\delta \Rightarrow\left|L_{k}(z, y)-L_{k}\left(z_{0}, y\right)\right|<\varepsilon$

Тогда функиия

$$
l(z)=\sup _{y \in Y}\left|f(y)+\sum_{k=1}^{m} L_{k}(z, y)\right|
$$

непрерывна на множестве $Z$.

ДоказАТЕльство. Само сушествование $l(z)$, очевидно, следует из условий 1 и 2 леммы. Пусть теперь $z_{0} \in Z$ произвольно и фиксировано, тогда

$$
l(z)=\sup _{y \in Y}\left|f(y)+\sum_{k=1}^{m} L_{k}\left(z_{0}, y\right)+r\left(z, z_{0}, y\right)\right|,
$$

где

$$
r\left(z, z_{0}, y\right)=\sum_{k=1}^{m}\left(L_{k}(z, y)-L_{k}\left(z_{0}, y\right)\right)
$$

Из (38) и свойств супремума следует, что

$$
l(z) \leqslant \sup _{y \in Y}\left|f(y)+\sum_{k=1}^{m} L_{k}\left(z_{0}, y\right)\right|+\sup _{y \in Y}\left|r\left(z, z_{0}, y\right)\right|=l\left(z_{0}\right)+\sup _{y \in Y}\left|r\left(z, z_{0}, y\right)\right| .
$$

С другой стороны, соотношение (39) и условие 3 леммы дают

$$
\sup _{y \in Y}\left|r\left(z, z_{0}, y\right)\right| \leqslant m \varepsilon
$$

для любого $z \in Z$ такого, что $\left|z-z_{0}\right|<\delta$. Значит,

$$
l(z)-l\left(z_{0}\right) \leqslant m \varepsilon
$$

Однако в силу равноправия $z$ и $z_{0}$ неравенство (42) может быть переписано:

$$
\left|l(z)-l\left(z_{0}\right)\right| \leqslant m \varepsilon
$$

откуда благодаря произвольности $\varepsilon$ вытекает непрерывность функции $l(z)$ в любой точке $z_{0} \in Z$.

ЗАмЕчАниЕ 1. Условие 2 леммы 1 сушественно и не вытекает из условия 3. Это показывает пример:

$$
m=1, \quad L(z, y)=z+y, \quad Y=Z=\mathbb{R}^{1}, \quad f(y) \equiv 0, \quad l(z)=+\infty .
$$


ЗАмечАниЕ 2. Условие 3 леммы 1 также сушественно. Это показывают примеры (здесь $Y=Z=\mathbb{R}^{1}$ ):

$$
\begin{array}{ll}
\text { 1) } f(y) \equiv 0, \quad m=1, & L(z, y)=1-e^{-z^{2} y^{2}} \\
\text { 2) } f(y) \equiv 0, \quad m=1, \quad L(z, y)=1-e^{-y^{2}\left(z^{2}-1+\left|z^{2}-1\right|\right)} .
\end{array}
$$

Обе эти функции непрерывны (даже равномерно-непрерывны) и ограничены на $\mathbb{R}^{2}$, однако функция $l(z)$, как легко видеть, в первом случае имеет разрыв при $z_{0}=0$, а во втором - разрывы при $z_{0}= \pm 1$.

ДОКАЗАТЕЛЬСТВО ТЕОРемЫ 1. ИЗ (12), (28), (26), (31), (33) и свойств супремума вытекает сушествование невязки $\Delta$, причем

$$
\begin{aligned}
\Delta \leqslant \Delta^{\prime}= & \sum_{i=1}^{2} \frac{\rho_{i}}{\pi^{\frac{3}{2}}} \int_{\mathbb{R}^{3}}\left[\sup _{(t, x) \in \mathbb{R}^{4}}\left|\frac{\partial \psi_{i}}{\partial t}+B_{i}(u, t, x)+A_{i}(u, t, x)\right|+\right. \\
& \left.+\sup _{(t, x) \in \mathbb{R}^{4}} A_{i}(u, t, x)\right] e^{-u^{2}} d u .
\end{aligned}
$$

Подставив (32) в (31) и (26) и введя обозначение

$$
\gamma=\left(\gamma_{1}, \gamma_{2}\right)=\left(\frac{1}{\sqrt{\beta_{1}}}, \frac{1}{\sqrt{\beta_{2}}}\right)
$$

получим

$$
\begin{aligned}
A_{i}(u, t, x)= & \psi_{1} \psi_{2} \rho_{j} \frac{d^{2}}{\sqrt{\pi}} \int_{\mathbb{R}^{3}} d w e^{-w^{2}} \mid \gamma_{i} u+\left(\bar{v}_{i}-\bar{v}_{j}\right)+ \\
& +s_{i} \gamma_{i}^{2}\left[\omega_{0 i} \times x\right]-s_{j} \gamma_{j}^{2}\left[\omega_{0 j} \times x\right]-\gamma_{j} w \mid, \\
B_{i}(u, t, x)= & \frac{\partial \psi_{i}}{\partial x}\left(\gamma_{i} u+\bar{v}_{i}+\gamma_{i}^{2} s_{i}\left[\omega_{0 i} \times x\right]\right)+ \\
& +2 \psi_{i} \gamma_{i} s_{i}\left\{\left(u,\left[\omega_{0 i} \times \bar{v}_{i}\right]\right)-s_{i} \gamma_{i}^{2}\left[\omega_{0 i} \times u\right] \cdot\left[\omega_{0 i} \times x\right]\right\} .
\end{aligned}
$$

Применим лемму 1 к каждому из супремумов, входяших в (44). Здесь $y=(t, x), Y=\mathbb{R}^{4}$, $z=(u, \gamma), Z=\mathbb{R}^{3} \times \mathbb{R}_{+}^{2}$, выполнение условий 1,2 леммы 1 вытекает из соотношений (33), (46), (47), а условие 3 удовлетворяется благодаря (33), полиномиальной структуре (47) относительно переменных $u$ и $\gamma$, а также равномерной сходимости интеграла (46) по $u$ и $\gamma$ на любом компакте, а по $x$ и $t$ - на всем пространстве $\mathbb{R}^{4}$. Тогда мы видим, что каждый из интегралов в (44) берется от функции, непрерывной по $u, \gamma$, и тоже, очевидно, сходится равномерно относительно $\gamma$ на любом компакте, а следовательно, вся величина $\Delta^{\prime}$ непрерьвна по $\gamma$ на $\mathbb{R}_{+}^{2}$. Значит, в (44) можно перейти к пределу при $\beta_{i} \rightarrow+\infty$, $i=1,2$, что равносильно устремлению $\gamma_{i}, i=1,2$, к нулю в (46) и (47). При этом, очевидно, зависимость от $t$ и $x$ остается лишь в функциях $\psi_{i}, i=1,2$, и их производных, а зависимость от $w$ и $u$ сводится лишь к выражениям $e^{-w^{2}}$ в (46) и $e^{-u^{2}}$ в (44). В результате тривиального интегрирования по $w$ и $u$ приходим к (35). 
ТЕОРема 2. Пусть выполнены все условия теоремы 1, за исключением (32), вместо которого предполагается, что

$$
\omega_{i}=\frac{\omega_{0 i} s_{i}}{\sqrt{\beta_{i}}}, \quad i=1,2
$$

Тогда справедлив следующий аналог утверждения (35):

$$
\begin{aligned}
\lim _{\substack{i \rightarrow+\infty \\
i=1,2}} \Delta^{\prime}= & \sum_{\substack{i, j=1 \\
i \neq j}}^{2} \rho_{i} \sup _{(t, x) \in \mathbb{R}^{4}}\left|\frac{\partial \psi_{i}}{\partial t}+\bar{v}_{i} \frac{\partial \psi_{i}}{\partial x}+\rho_{j} \pi d^{2} \psi_{1} \psi_{2}\right| \bar{v}_{i}-\bar{v}_{j}||+ \\
& +2 \pi d^{2} \rho_{1} \rho_{2}\left|\bar{v}_{1}-\bar{v}_{2}\right| \sup _{(t, x) \in \mathbb{R}^{4}}\left(\psi_{1} \psi_{2}\right)+\frac{4}{\sqrt{\pi}} \sum_{i=1}^{2} \rho_{i} s_{i}\left|\left[\omega_{0 i} \times \bar{v}_{i}\right]\right| \sup _{(t, x) \in \mathbb{R}^{4}} \psi_{i} .
\end{aligned}
$$

ДоКАЗАТЕЛЬСтво. Воспользуемся оценкой (44), не вводя пока обозначения $\Delta^{\prime}$ для ее правой части. В (31) и (26) подставим (48), вновь используя обозначение (45). Тогда вместо выражений (46), (47) получим

$$
\begin{aligned}
A_{i}(u, t, x)= & \psi_{1} \psi_{2} \rho_{j} \frac{d^{2}}{\sqrt{\pi}} \int_{\mathbb{R}^{3}} d w e^{-w^{2}} \mid \gamma_{i} u+\left(\bar{v}_{i}-\bar{v}_{j}\right)+ \\
& +s_{i} \gamma_{i}\left[\omega_{0 i} \times x\right]-s_{j} \gamma_{j}\left[\omega_{0 j} \times x\right]-\gamma_{j} w \mid, \\
B_{i}(u, t, x)= & \frac{\partial \psi_{i}}{\partial x}\left(\gamma_{i} u+\bar{v}_{i}+s_{i} \gamma_{i}\left[\omega_{0 i} \times x\right]\right)+ \\
& +2 \psi_{i} s_{i}\left\{\left(u,\left[\omega_{0 i} \times \bar{v}_{i}\right]\right)-s_{i} \gamma_{i}\left[\omega_{0 i} \times u\right] \cdot\left[\omega_{0 i} \times x\right]\right\} .
\end{aligned}
$$

После подстановки (50), (51) в (44) оценим полученное выражение сверху:

$$
\begin{aligned}
\Delta \leqslant \Delta^{\prime}= & \sum_{i=1}^{2} \frac{\rho_{i}}{\pi^{\frac{3}{2}}} \int_{\mathbb{R}^{3}}\left[\sup _{(t, x) \in \mathbb{R}^{4}} \mid \frac{\partial \psi_{i}}{\partial t}+A_{i}(u, t, x)+\right. \\
& +\frac{\partial \psi_{i}}{\partial x}\left(\gamma_{i} u+\bar{v}_{i}+s_{i} \gamma_{i}\left[\omega_{0 i} \times x\right]\right)-2 \psi_{i} s_{i}^{2} \gamma_{i}\left[\omega_{0 i} \times u\right] \cdot\left[\omega_{0 i} \times x\right] \mid+ \\
& \left.+\sup _{(t, x) \in \mathbb{R}^{4}} A_{i}(u, t, x)+\sup _{(t, x) \in \mathbb{R}^{4}} 2 \psi_{i} s_{i}|u|\left|\left[\omega_{0 i} \times \bar{v}_{i}\right]\right|\right] e^{-u^{2}} d u
\end{aligned}
$$

Применение леммы 1 к каждому из супремумов в (52) (обоснование ее применимости совершенно аналогично проведенному при доказательстве теоремы 1 , причем третье слагаемое под интегралом в (52) вообще не зависит от $\beta_{i}$, т.е. от $\gamma$ ) позволяет перейти в (52) к пределу при $\beta_{i} \rightarrow+\infty, i=1,2$. При этом, очевидно, результат будет отличаться от (35) лишь упомянутым третьим слагаемым, которое тривиально вычисляется, что и приводит к (49). Теорема доказана.

Следуюшие две теоремы относятся к частному случаю, когда $x_{0 i}=0, i=1,2$, т.е. оси врашения обоих винтов проходят через начало координат.

TEOPEMA 3. Пусmb

$$
\omega_{i}=\frac{\omega_{0 i} s_{i}}{\sqrt[4]{\beta_{i}}}, \quad i=1,2
$$


причем

$$
\left[\omega_{0 i} \times \bar{v}_{i}\right]=0 .
$$

Тогда при выполнении условий (33) справедливо (34), где

$$
\begin{aligned}
\lim _{\substack{\beta_{i} \rightarrow+\infty \\
i=1,2}} \Delta^{\prime}= & \sum_{\substack{i, j=1 \\
i \neq j}}^{2} \rho_{i} \sup _{(t, x) \in \mathbb{R}^{4}}\left|\frac{\partial \psi_{i}}{\partial t}+\bar{v}_{i} \frac{\partial \psi_{i}}{\partial x}+\rho_{j} \pi d^{2} \psi_{1} \psi_{2}\right| \bar{v}_{i}-\bar{v}_{j}||+ \\
& +2 \pi d^{2} \rho_{1} \rho_{2}\left|\bar{v}_{1}-\bar{v}_{2}\right| \sup _{(t, x) \in \mathbb{R}^{4}}\left(\psi_{1} \psi_{2}\right)+ \\
& +\frac{4}{\sqrt{\pi}} \sum_{i=1}^{2} \rho_{i} s_{i}^{2}\left|\omega_{0 i}\right| \sup _{(t, x) \in \mathbb{R}^{4}}\left(\left|\left[\omega_{0 i} \times x\right]\right| \psi_{i}\right) .
\end{aligned}
$$

ДокАЗАТЕЛЬСТво проводится по схеме доказательства теоремы 2, однако теперь благодаря (53), (54) вместо (50), (51) из (31), (26) имеем

$$
\begin{aligned}
A_{i}(u, t, x)= & \psi_{1} \psi_{2} \rho_{j} \frac{d^{2}}{\sqrt{\pi}} \int_{\mathbb{R}^{3}} d w e^{-w^{2}} \mid \gamma_{i} u+\left(\bar{v}_{i}-\bar{v}_{j}\right)+ \\
& +\sqrt{\gamma_{i}} s_{i}\left[\omega_{0 i} \times x\right]-\sqrt{\gamma_{j}} s_{j}\left[\omega_{0 j} \times x\right]-\gamma_{j} w \mid, \\
B_{i}(u, t, x)= & \frac{\partial \psi_{i}}{\partial x}\left(\gamma_{i} u+\bar{v}_{i}+\sqrt{\gamma_{i}} s_{i}\left[\omega_{0 i} \times x\right]\right)-2 \psi_{i} s_{i}^{2}\left[\omega_{0 i} \times u\right] \cdot\left[\omega_{0 i} \times x\right] .
\end{aligned}
$$

Как видно из $(56),(57)$, предел при $\gamma \rightarrow 0$ величины $A_{i}(u, t, x)$ останется таким же, как в теоремах 1,2 , а оценка сверху для модуля, входящего в $(28)$ и содержащего $B_{i}(u, t, x)$, подобная оценке (52), приведет теперь к выделению не зависящего от $\gamma$ (т.е. от $\beta_{i}, i=$ $1,2)$ слагаемого, в которое войдут выражения

$$
2 s_{i}^{2} \int_{\mathbb{R}^{3}}\left|\psi_{i}\left[\omega_{0 i} \times u\right] \cdot\left[\omega_{0 i} \times x\right]\right| \frac{\rho_{i}}{\pi^{\frac{3}{2}}} e^{-u^{2}} d u, \quad i=1,2 .
$$

Дальнейшая очевидная оценка сверху для (58) ввиду выполнения (33) приводит к (55). Теорема доказана.

В формулировке следующей теоремы вообще не участвуют функции $\psi_{i}, i=1,2$, т.е. не используется предположение (24).

ТЕОРема 4. Пусть распределение $f$ имеет вид(9)-(11), где функции $\varphi_{i}, i=1,2$, не зависят от $\beta_{i}, i=1,2$, причем выполнено одно из условий (32) или (48), и, кроме того, имеет место (54). Положим

$$
\mu_{i}= \begin{cases}1, & \text { если верно }(32), \\ e^{s_{i}^{2}\left[\omega_{0 i} \times x\right]^{2}}, & \text { если верно (48). }\end{cases}
$$

Тогда если величинь

$$
\begin{gathered}
\varphi_{i} e^{\beta_{i} \omega_{i}^{2} r_{i}^{2}}, \quad \frac{\partial \varphi_{i}}{\partial t} e^{\beta_{i} \omega_{i}^{2} r_{i}^{2}}, \quad\left|\frac{\partial \varphi_{i}}{\partial x}\right| e^{\beta_{i} \omega_{i}^{2} r_{i}^{2}}, \\
\varphi_{i}\left|\left[\omega_{0 i} \times x\right]\right| e^{\beta_{i} \omega_{i}^{2} r_{i}^{2}}, \quad\left|\frac{\partial \varphi_{i}}{\partial x}\left[\omega_{0 i} \times x\right]\right| e^{\beta_{i} \omega_{i}^{2} r_{i}^{2}},
\end{gathered}
$$


ограниченьг по $t, x$ на $\mathbb{R}^{4}$ при $\beta_{i} \rightarrow+\infty, i=1,2$, то справедливо (34), причем

$$
\begin{aligned}
\lim _{\substack{\beta_{i} \rightarrow+\infty \\
i=1,2}} \Delta^{\prime}= & \sum_{\substack{i, j=1 \\
i \neq j}}^{2} \rho_{i} \sup _{(t, x) \in \mathbb{R}^{4}} \mid\left(\frac{\partial \varphi_{i}}{\partial t}+\bar{v}_{i} \frac{\partial \varphi_{i}}{\partial x}\right) \mu_{i}(x)+ \\
& +\varphi_{1} \varphi_{2} \mu_{1}(x) \mu_{2}(x) \pi d^{2} \rho_{j}\left|\bar{v}_{i}-\bar{v}_{j}\right| \mid+ \\
& +2 \rho_{1} \rho_{2} \pi d^{2}\left|\bar{v}_{1}-\bar{v}_{2}\right| \sup _{(t, x) \in \mathbb{R}^{4}}\left[\mu_{1}(x) \mu_{2}(x) \varphi_{1} \varphi_{2}\right] .
\end{aligned}
$$

ДокАЗАТЕЛЬство. Условия (60), как видно из (18), (19), (23), обеспечивают существование невязки $\Delta$. Из (6), (7) и (59) с учетом (54) получаем, что при выполнении $(32)$ или (48) экспоненциальные факторы в (23) ведут себя следуюшим образом:

$$
\lim _{\substack{\beta_{i} \rightarrow+\infty \\ i=1,2}} e^{\beta_{i} \omega_{i}^{2} r_{i}^{2}}=\mu_{i}(x), \quad i=1,2 .
$$

Тогда, производя оценку сверху для $\Delta$ так же, как это делалось при доказательстве теоремы 1 , и обозначая ее через $\Delta^{\prime}$, а затем вновь опираясь на лемму 1 , очевидно, придем к (61).

ЗАмЕчАНИЕ 3. В каждой из теорем 1-4 предполагается, что обе угловые скорости винтов $\omega_{1}$ и $\omega_{2}$ стремятся к нулю при $\beta_{1}, \beta_{2} \rightarrow+\infty$ одинаково быстро (сама скорость их стремления к нулю различна и задается либо (32), либо (48), либо (53)). Разумеется, можно рассматривать и такие варианты, когда $\omega_{1}$ и $\omega_{2}$ ведут себя по-разному. Как видно из доказательств теорем 1-4, это приведет к “гибридным" вариантам предельного выражения для $\Delta^{\prime}$, в которых при $i=1$ и $i=2$ будут участвовать различные слагаемые, встречающиеся в каждой из формул (35), (49), (55), (61).

До сих пор мы полагали величины $\bar{v}_{i}, i=1,2$, фиксированными. Теперь приведем один из результатов, относящихся к случаю, когда $\omega_{i}$ и $\bar{v}_{i}, i=1,2$, стремятся к нулю одновременно и согласованно, т.е. по некоторой “траектории” в пространстве параметров.

ТЕОрема 5. Пусть выполнено условие (53) и, кроме того,

$$
\bar{v}_{i}=\frac{\sigma_{i} \bar{v}_{0 i}}{\sqrt[4]{\beta_{i}}}, \quad i=1,2,
$$

где $\sigma_{i}>0$ - любие постоянные, $\bar{v}_{0 i}$ - произвольные фиксированные векторы. Тогда при выполнении (33) справедливо (34), где

$$
\begin{gathered}
\lim _{\substack{\beta_{i} \rightarrow+\infty \\
i=1,2}} \Delta^{\prime}=\sum_{i=1}^{2} \rho_{i}\left[\sup _{(t, x) \in \mathbb{R}^{4}}\left|\frac{\partial \psi_{i}}{\partial t}\right|+\frac{4}{\sqrt{\pi}} s_{i}^{2}\left|\omega_{0 i}\right| \sup _{(t, x) \in \mathbb{R}^{4}}\left(\left|\left[\omega_{0 i} \times x\right]\right| \psi_{i}\right)+\right. \\
\left.+\frac{4}{\sqrt{\pi}} s_{i} \sigma_{i}\left|\left[\omega_{0 i} \times \bar{v}_{0 i}\right]\right| \sup _{(t, x) \in \mathbb{R}^{4}} \psi_{i}\right] .
\end{gathered}
$$


ДоКАЗАТЕЛЬСТВо аналогично доказательству теоремы 3 , однако ввиду отсутствия в теореме 5 условия (54) и наличия условия (63) вместо (56), (57) из соотношений (31), (26) теперь имеем

$$
\begin{aligned}
A_{i}(u, t, x)= & \psi_{1} \psi_{2} \rho_{j} \frac{d^{2}}{\sqrt{\pi}} \int_{\mathbb{R}^{3}} d w e^{-w^{2}} \mid \gamma_{i} u+\sigma_{i} \sqrt{\gamma_{i}} \bar{v}_{0 i}- \\
& -\sigma_{j} \sqrt{\gamma_{j}} \bar{v}_{0 j}+\sqrt{\gamma_{i}} s_{i}\left[\omega_{0 i} \times x\right]-\sqrt{\gamma_{j}} s_{j}\left[\omega_{0 j} \times x\right]-\gamma_{j} w \mid, \\
B_{i}(u, t, x)= & \frac{\partial \psi_{i}}{\partial x}\left(\gamma_{i} u+\sigma_{i} \sqrt{\gamma_{i}} \bar{v}_{0 i}+\sqrt{\gamma_{i}} s_{i}\left[\omega_{0 i} \times x\right]\right)- \\
& -2 \psi_{i} s_{i}^{2}\left[\omega_{0 i} \times u\right] \cdot\left[\omega_{0 i} \times x\right]+2 \psi_{i} s_{i} \sigma_{i}\left(u,\left[\omega_{0 i} \times \bar{v}_{0 i}\right]\right) .
\end{aligned}
$$

Очевидно, что вклад от $A_{i}(u, t, x)(65)$ в пределе при $\gamma_{i} \rightarrow 0, i=1,2$, исчезает, а оценка сверху величин, содержащих $B_{i}(u, t, x)(66)$, приведет к выделению двух не зависящих от $\gamma_{i}$ слагаемых, соответственно аналогичных (58) и последнему члену в (52) (с заменой $\bar{v}_{i}$ на $\left.\sigma_{i} \bar{v}_{0 i}\right)$, что и влечет $(64)$.

ЗАмЕчАНИЕ 4. Можно вместо (63) рассмотреть и иные варианты поведения $\bar{v}_{i}, i=$ 1,2 , аналогичные (32) или (48). В сочетании с тремя рассмотренными в теоремах $1-3$ вариантами поведения $\omega_{i}, i=1,2$, это приводит к большому количеству различных ситуаций. Однако их анализ показывает, что предел величины $\Delta^{\prime}$ всегда будет описываться одной из формул $(35),(49),(55),(61),(64)$. На этом пути можно получить и некоторые аналоги теоремы 4 , с той лишь разницей, что в роли $\mu_{i}(x), i=1,2$, будут выступать несколько иные выражения, чем (59), а в (61) следует положить $\bar{v}_{1}=\bar{v}_{2}=0$. Отметим также, что в тех случаях, когда скорости стремления $\omega_{i}$ и $\bar{v}_{i}$ к нулю различны, вектор $x_{0 i}$, определенный в $(6)$, стремится либо к нулю, либо к бесконечности, в то время как при одинаковом порядке малости $\omega_{i}$ и $\bar{v}_{i}$ вектор $x_{0 i}$ "стабилизируется", т.е. стремится к конечному значению

$$
\frac{\sigma_{i}}{s_{i}} \cdot \frac{\left[\omega_{0 i} \times \bar{v}_{0 i}\right]}{\omega_{0 i}^{2}}
$$

Как видно из предыдущих рассуждений, такое поведение вектора $x_{0 i}$ невозможно, если $\bar{v}_{i}$ фиксировано, как это было в теоремах 1-4 (там вектор $x_{0 i}$ либо стремится к бесконечности, либо просто равен нулю в случае, когда $\bar{v}_{i} \| \omega_{i}$, т.е. выполнено (54)).

ЗАмечАниЕ 5. В теоремах 1-5 и замечании 4 речь идет только о таких ситуациях, когда векторы $\omega_{i}, \bar{v}_{i}, i=1,2$, стремятся к нулю обратно пропорционально некоторым избранным степеням величин $\beta_{i}, i=1,2$, а именно: $1,1 / 2$ либо $1 / 4$ (кроме того, вектор $\bar{v}_{i}$ может быть просто постоянным). Выбор именно таких значений продиктован, в первую очередь, структурой выражения (31). Нетрудно убедиться в том, что при любых иных скоростях стремления этих векторов к нулю в поведении невязки $\Delta$ и вектора $x_{0 i}$ не появляется ничего нового по сравнению с рассмотренными выше вариантами.

ЗАмЕЧАНИЕ 6. Возможны и некоторые более “экзотические” варианты поведения векторов $\omega_{i}, \bar{v}_{i}, i=1,2$, приводящие к любопытным эффектам. Например, если поло- 
жить

$$
\omega_{i}=\frac{s_{i} \omega_{0 i}}{\beta_{i}}, \quad \bar{v}_{i}=\frac{\sigma_{i} \bar{v}_{0 i}}{\beta_{i}} \sin \beta_{i}, \quad i=1,2,
$$

то окажется, что невязка $\Delta^{\prime}$ по-прежнему имеет конечный предел, а именно

$$
\lim _{\substack{\beta_{i} \rightarrow+\infty \\ i=1,2}} \Delta^{\prime}=\sum_{i=1}^{2} \rho_{i} \sup _{(t, x) \in \mathbb{R}^{4}}\left|\frac{\partial \varphi_{i}}{\partial t}\right|
$$

в то время как векторы $x_{0 i}$ не будут иметь ни конечного, ни бесконечного предела, но будут "осциллировать" по закону

$$
x_{0 i}(\beta)=\frac{\sigma_{i}}{s_{i}} \cdot \frac{\left[\omega_{0 i} \times \bar{v}_{0 i}\right]}{\omega_{0 i}^{2}} \sin \beta_{i} .
$$

ЗАмЕЧАниЕ 7. Наконец, можно, так же как в замечании 3 , варьировать поведение не только векторов $\omega_{i}$, но и $\bar{v}_{i}$, в зависимости от индекса $i$. Это резко увеличивает формально возможное количество вариантов поведения невязки, приводя к появлению смешанных выражений, однако не дает ничего принципиально нового, ибо при каждом $i$ отдельно никаких иных эффектов, кроме описанных выше, обнаружить не удается.

\section{3. МИНИМИЗАЦИЯ НЕВЯЗКИ}

Опираясь на полученные в разделе 2 выражения для пределов при $\beta_{i} \rightarrow+\infty, i=1,2$, можно найти некоторые достаточные условия стремления невязки $\Delta$ к нулю, которые удобно оформить в виде следствий из теорем 1-5 (во всех дальнейших формулировках предполагается, что указанный предельный переход уже осушествлен). Подробных доказательств следствий, как правило, приводить не будем, ибо некоторые из них очевидны, а другие повторяют аналогичные рассмотрения работы [18].

СЛЕДСТВИЕ 1. Пусть выполнены все предположсения теоремы 1. Тогда соотношение

$$
\Delta \rightarrow 0
$$

справедливо, если имеет место хотя бъ одно из условий:

1) для любъх функций $\psi_{i}(x)$, удовлетворяющих условиям (33),

$$
\bar{v}_{1}=\bar{v}_{2}=0, \quad \psi_{i}=\psi_{i}(x), \quad i=1,2 ;
$$

2) $\bar{v}_{1}=\bar{v}_{2} \neq 0$, вьполнено (54) $и$

$$
\psi_{i}=C_{i}\left(\left[x \times \bar{v}_{i}\right]\right), \quad i=1,2,
$$

әде $C_{i} \geqslant 0$ - любъе гладкие финитные либо быстроубывающие функиии своих векторных аргументов;

3) $\bar{v}_{1}=\bar{v}_{2} \neq 0$, выполнено (54) и

$$
\psi_{i}=C_{i}\left(x-\bar{v}_{i} t\right), \quad i=1,2,
$$


әде $C_{i}$ такие же, как в пункте 2 ;

4) $\bar{v}_{1}=0$, векторь $\bar{v}_{2}, \omega_{01}, \omega_{02}$ коллинеарнь,

$$
\begin{aligned}
& \psi_{1}=\psi_{1}(t, x)=h\left(\left[x \times \bar{v}_{2}\right]\right)\left\{\lambda+C\left(\left[x \times \bar{v}_{2}\right]\right) \times\right. \\
&\left.\times \exp \left[-\pi d^{2}\left|\bar{v}_{2}\right| h\left(\left[x \times \bar{v}_{2}\right]\right)\left(\frac{x^{1}}{\bar{v}_{2}^{1}}\left(\frac{\rho_{2}}{\mu}+\frac{\rho_{1}}{\lambda}\right)-\frac{\rho_{2}}{\mu} t\right)\right]\right\}^{-1}, \\
& \psi_{2}=\psi_{2}(t, x)=\frac{1}{\mu}\left(h\left(\left[x \times \bar{v}_{2}\right]\right)-\lambda \psi_{1}(t, x)\right)
\end{aligned}
$$

где $\lambda, \mu>0$ - произвольные константы, а функции $h$ и $C$ имеют такие же свойства, как $C_{i}, i=1,2$, в (73), и, кроме того,

$$
d \rightarrow 0
$$

5) $\bar{v}_{1} \neq 0, \quad \bar{v}_{2} \neq 0$ произвольньи, выполнено (54), функции $\psi_{i}, \quad i=1,2$, имеют вид (74) и, кроме того,

$$
\operatorname{supp} \psi_{1} \cap \operatorname{supp} \psi_{2}=\varnothing ;
$$

6) $\bar{v}_{1} \neq 0, \bar{v}_{2} \neq 0$ произвольны, функции $\psi_{i}, \quad i=1,2$, имеют вид (73) либо (74) и вьгполнень (77) $u(54)$.

ДокАзАТЕЛьство опирается на формулы (34), (35), на результаты работы [18], где, в частности, показано, что функции $(75),(76)$ при указанных в пункте 4 условиях являются одним из решений системы дифференщиальных уравнений

$$
\frac{\partial \psi_{i}}{\partial t}+\bar{v}_{i} \frac{\partial \psi_{i}}{\partial x}=-\rho_{j} \pi d^{2} \psi_{1} \psi_{2}\left|\bar{v}_{2}\right|, \quad i, j=1,2, \quad i \neq j
$$

и на проверку того, что условия (72)-(76) удовлетворяют ограниченности величин (33).

СЛЕДСТВИЕ 2. Пусть имеют место все предположсения теоремы 2. Тогда справедливо (71), если выполнены хотя бъ одно из условий следствия 1 и, кроме того, хотя бы одно из следующих требований:

1) $s_{i} \rightarrow 0, \quad i=1,2$;

2) условие (54);

3) $s_{i} \rightarrow 0, \omega_{0 j} \| \bar{v}_{j}, \quad i, j=1,2, \quad i \neq j$.

ДоКАЗАТЕЛЬСтво очевидно и опирается на (49).

СЛЕДСТВИЕ 3. Пусть имеют место все предположсения теоремы 3. Тогда справедливо (71), если выполнены хотя бы одно из условий следствия 1 и, кроме того, требование 1 следствия 2.

ДокАЗАТЕЛЬСТво тривиально проводится на основании (55). 
ЛЕмма 2. При выполнении условий теоремъ 4 замена функций

$$
\xi_{i}=\mu_{i} \varphi_{i}, \quad i=1,2,
$$

приводит правую часть (61) к виду (35), где вместо $\psi_{i}, \quad i=1,2$, фигурируют функции $\xi_{i}$.

ДоказАтельство. Если выполнено (32), то, как видно из (59), $\mu_{i}=1, i=1,2$, и утверждение леммы тривиально. Пусть теперь имеет место (48), тогда из (80) и (59) получим

$$
\varphi_{i}=\xi_{i} e^{-s_{i}^{2}\left[\omega_{0 i} \times x\right]^{2}}, \quad i=1,2
$$

Отсюда

$$
\begin{aligned}
& \frac{\partial \varphi_{i}}{\partial t}=\frac{\partial \xi_{i}}{\partial t} e^{-s_{i}^{2}\left[\omega_{0 i} \times x\right]^{2}} \\
& \frac{\partial \varphi_{i}}{\partial x}=e^{-s_{i}^{2}\left[\omega_{0 i} \times x\right]^{2}}\left\{\frac{\partial \xi_{i}}{\partial x}-2 \xi_{i} s_{i}^{2}\left[\left[\omega_{0 i} \times x\right] \times \omega_{0 i}\right]\right\} .
\end{aligned}
$$

Подставляя (81)-(83) в (61), имеем

$$
\begin{aligned}
\lim _{\substack{\beta_{i} \rightarrow \infty \\
i=1,2}} \Delta^{\prime}= & \sum_{\substack{i, j=1 \\
i \neq j}}^{2} \rho_{i} \sup _{(t, x) \in \mathbb{R}^{4}} \mid \frac{\partial \xi_{i}}{\partial t}+\bar{v}_{i} \frac{\partial \xi_{i}}{\partial x}-2 \xi_{i} s_{i}^{2}\left(\bar{v}_{i},\left[\omega_{0 i} \times x\right], \omega_{0 i}\right)+ \\
& +\xi_{1} \xi_{2} \pi d^{2} \rho_{j}\left|\bar{v}_{i}-\bar{v}_{j}\right|\left|+2 \rho_{1} \rho_{2} \pi d^{2}\right| \bar{v}_{1}-\bar{v}_{2} \mid \sup _{(t, x) \in \mathbb{R}^{4}}\left(\xi_{1} \xi_{2}\right)
\end{aligned}
$$

Однако в силу (54) смешанное произведение в третьем слагаемом (84) равно нулю, т.е. выражение (84) совпадает с (35) при замене $\psi_{i}$ на $\xi_{i}, i=1,2$, что и требовалось доказать.

ЗАмЕчаниЕ 8. При “смешанном" варианте замены типа (80), когда $\mu_{i}=1, \mu_{j}=$ $e^{s_{j}^{2}\left[\omega_{0 j} \times x\right]^{2}}, i=1,2, i \neq j$, как нетрудно убедиться, утверждение леммы 2 также остается в силе.

СлЕДСТВИЕ 4. Пусть выполнены все предположения теоремы 4 и, кроме того, функиии $\varphi_{i} e^{\beta_{i} \omega_{i}^{2} r_{i}^{2}}\left|\left[\omega_{0 i} \times x\right]\right|^{2}$ ограничены по $t, x$ на $\mathbb{R}^{4}$ nри $\beta_{i} \rightarrow+\infty, i=1,2$. Тогда, если $\varphi_{i}$ таковы, что функиии $\xi_{i}$, определенные в (80), (59), удовлетворяют одному из условий 1-6, которье накладьваются на функиии $\psi_{i}$ в следствии 1, то имеет место (71).

ДокАЗАТЕЛьство. В силу леммы 2 выражение (61) с участием функций $\varphi_{i}, i=1,2$, при замене (80) формально переходит в (35) для функций $\xi_{i}, i=1,2$. Поэтому чтобы с помощью следствия 1 получить (71), остается проверить, что вьполнение условий 
следствия 4 для функций $\varphi_{i}$ влечет за собой выполнение условий теоремы 1 (т.е. требований (33)) для функций $\xi_{i}$. С этой целью продифференцируем (80) по $t$ и $x$ :

$$
\begin{aligned}
\frac{\partial \xi_{i}}{\partial t} & =\frac{\partial \varphi_{i}}{\partial t} \mu_{i}, \\
\frac{\partial \xi_{i}}{\partial x} & =\frac{\partial \varphi_{i}}{\partial x} \mu_{i}+\varphi_{i} \frac{\partial \mu_{i}}{\partial x}
\end{aligned}
$$

где, как легко видеть из (59),

$$
\frac{\partial \mu_{i}}{\partial x}= \begin{cases}0, & \text { если справедливо (32), } \\ e^{s_{i}^{2}\left[\omega_{0 i} \times x\right]^{2}} 2 s_{i}^{2}\left[\left[\omega_{0 i} \times x\right] \times \omega_{0 i}\right], & \text { если справедливо (48). }\end{cases}
$$

Далее, переходя к пределу при $\beta_{i} \rightarrow+\infty, i=1,2$, в каждом из выражений (60) и в выражениях $\varphi_{i} e^{\beta_{i} \omega_{i}^{2} r_{i}^{2}}\left|\left[\omega_{0 i} \times x\right]\right|^{2}$, с учетом $(62)$ и условия следствия 4 видим, что величины

$$
\varphi_{i} \mu_{i}, \quad \frac{\partial \varphi_{i}}{\partial t} \mu_{i}, \quad\left|\frac{\partial \varphi_{i}}{\partial x}\right| \mu_{i}, \quad \varphi_{i} \mu_{i}\left|\left[\omega_{0 i} \times x\right]\right|, \quad \mu_{i}\left|\left[\omega_{0 i} \times x\right] \frac{\partial \varphi_{i}}{\partial x}\right|, \quad \varphi_{i} \mu_{i}\left|\left[\omega_{0 i} \times x\right]\right|^{2}
$$

ограничены на $\mathbb{R}^{4}$. Тогда из (85)-(87) следует, что для функций $\xi_{i}, i=1,2$, выполнено (33).

СЛЕДСТВИЕ 5. Пусть имеют место все предположения теоремы 5. Тогда справедливо (71), если выполняются условия (72) и требование 1 следствия 2.

ДокАЗАТЕЛЬСТво. Справедливость (71) непосредственно вытекает из (64), (72) и требования 1 следствия 2 .

\section{4. ОБСУЖДЕНИЕ РЕЗУЛЬТАТОВ}

Проанализируем теперь полученные результаты с точки зрения их физического смысла. Сразу же отметим, что точными решениями уравнения Больцмана, имеющими хорошо известный физический смысл, являются сами винтообразные максвелловские распределения (10), (11). А бимодальные распределения вида (9), которые рассмотрены нами в данной работе, лишь приближенно описывают взаимодействие таких винтов и удовлетворяют уравнению Больцмана также приближенно, в смысле минимизации невязки $\Delta$. Тем не менее они могут быть разумно истолкованы физически.

Общим свойством всех найденных распределений является то, что они описывают неравномерно остываюший газ $\left(\beta_{i} \rightarrow+\infty, i=1,2\right)$, причем вращение обоих винтов замедляется $\left(\omega_{i} \rightarrow 0, i=1,2\right)$, хотя и в различной степени, в соответствии с $(32),(48)$ или (53) (в некоторых случаях, кроме того, предполагается выполненным требование 1 следствия 2). При этом само распределение $f$ не стремится ни к одному из максвеллианов (т.е. к известному точному решению уравнения Больцмана), ибо уже на первом этапе, когда $\beta_{i} \rightarrow+\infty, i=1,2$, выражения (10) не имеют предела в смысле пространства $L_{1}$ по переменной $v$, в то время как интеграл в (12) имеет такой предел. 
Напомним, что условие (77) соответствует "околосвободномолекулярному" течению (газ, близкий к кнудсеновскому [1]), а условие $x_{0 i}=0$, равносильное (54), означает, что $\bar{v}_{i} \| \omega_{0 i}$ и ось врашения винта проходит через начало координат $O$. Условие $x_{0 i} \rightarrow \infty$ показывает, что винты неограниченно удаляются друг от друга, а случаи, когда $x_{0 i}$ стремится к ненулевой константе или вообше не имеет предела (см. замечания 4, 6), соответствуют тому, что оси винтов не пересекаются между собой и либо “стабилизируются" на конечном расстоянии друг от друга, либо “осциллируют". Далее, выполнение условия (78) соответствует стратификации объектов ("сгустков газа") в $\mathbb{R}^{4}$. Наконец, условия $\bar{v}_{i}=0$ либо $\bar{v}_{i} \rightarrow 0, i=1,2$, означают, что $i$-й винт врашается вокруг своей оси, не совершая при этом поступательного движения вдоль нее (или замедляя это движение).

С учетом всего вышесказанного можно выделить следуюшие ситуации, обеспечивающие выполнение (71).

1. Большие числа Кнудсена $(d \rightarrow 0)$ :

а) оси винтов совпадают, один из них покоится, другой движется вдоль общей оси, их плотности пропорциональны (75), (76);

б) стационарные вращающиеся цилиндры с осями, пересекающимися в точке $O$, массовые скорости вдоль осей произвольны, плотности задаются в (73);

в) нестационарные вращающиеся сгустки газа, движушиеся с произвольными скоростями через точку $O$, плотности описываются в (74).

2. Произвольные числа Кнудсена (любое $d>0$ ):

а) оба винта покоятся, оси проходят через точку $O$, плотности стационарны и произвольны (заданы в (72));

б) коаксиальные стационарные винты с произвольными равными линейными скоростями, плотности задаются в (73);

в) коаксиальные врашающиеся сгустки (нестационарные винты), движущиеся с одинаковыми скоростями через точку $O$, плотности описаны в (74);

г) врашаюшиеся сгустки с произвольными скоростями и осями, пересекаюшимися в точке $O$, не сталкиваюшиеся между собой (в силу (78)), плотности заданы в (74).

ЗАмЕчаниЕ 9. Во всех рассмотренных выше случаях, кроме описанных в теореме 4 и следствии 4 , плотности винтов вообше не зависят от $\beta_{i}, i=1,2$, а в теореме 4 и следствии 4 упомянутая зависимость есть, однако она такова, что при $\beta_{i} \rightarrow+\infty$ плотности имеют конечные пределы (а именно, $\left.\rho_{i} \xi_{i}, i=1,2\right)$. Как видно из $(32),(48),(54)$, это возможно лишш в тех случаях, когда оси обоих винтов проходят через точку $O$, а их вращение замедляется достаточно быстро.

ЗАмЕчАниЕ 10. Наконец, возможны и некоторые "смешанные" варианты поведения объектов при различных $i=1,2$ (например, один из объектов может быть стационарным цилиндром и описываться соотношением (73), а другой- нестационарным сгустком вида (74)).

Итак, нам удалось построить целый ряд бимодальных распределений вида (9)-(11), приближенно удовлетворяющих уравнению Больцмана (1)-(4) в смысле минимизации 
невязки (12). Возможно, не все такие распределения были нами обнаружены, что связано с неоднозначностью оценок сверху типа (18). Однако пока не удается найти предел при $\beta_{i} \rightarrow+\infty$ самого выражения невязки $\Delta$ для распределений вида $(9)-(11)$, подобно тому как это было сделано в работе [16] для функции $f$ значительно более простого вида. Кроме того, интересно было бы рассмотреть случай, когда сами локальные максвеллианы нестационарны, т.е. обобшить полученные результаты на распределения более обшего, чем (5), вида.

\section{Список литературы}

[1] K. Черчинълни. Теория и приложения уравнения Больцмана. М.: Мир, 1978.

[2] Т. Карлеман. Математические задачи кинетической теории газов. М.: ИИЛ, 1960.

[3] R.S. Krupp. Magister Sciences Thesis. MiT, 1967.

[4] А. В. Бобылев. ДАН СССР. 1975. Т. 225. № 6. С. 1296.

[5] А. В. Бобылев. ДАН СССР. 1976. Т. 231. № 3. С. 571.

[6] M. Krook, T. Wu. Phys. Fluids. 1977. V. 20. № 10(1). P. 1589.

[7] А. А. Николъский. ДАН СССР. 1963. Т. 151. № 2. С. 299.

[8] E. Ikenberry, C. Truesdall. Ration. Mech. Anal. 1956. V. 5. № 1. P. 1.

[9] В. С. Галкин. Прикл. матем. и мех. 1956. Т. 20. № 3. С. 445.

[10] H. M. Ernst. J. Stat. Phys. 1984. V. 34. № 5/6. P. 1001.

[11] А. В. Бобылев. ДАН СССР. 1981. Т. 256. № 6. С. 1341.

[12] В. В. Веденяпин. ДАН СССР. 1981. Т. 256. № 2. С. 338.

[13] Д. Я. Петрина, А. В. Мищенко. ДАН СССР. 1988. Т. 298. № 2. С. 338.

[14] А. В. Мищенко, Д. Я. Петрина. ТМФ. 1988. Т. 77. № 1. С. 135.

[15] В. Д. Гордевский. Матем. физ., анализ, геом. 1995. Т. 2. № 2. С. 168.

[16] В. Д. Гордевский. Матем. физ., анализ, геом. 1997. Т. 4. № 1/2. С. 46.

[17] В. Д. Гордевский. ТМФ. 1998. Т. 114. № 1. С. 126.

[18] В.Д. Гордевский. Некоторые классы приближенных бимодальных решений нелинейного уравнения Больцмана. В сб.: Интегральные преобразования и их применение к краевым задачам. Ред. М. П. Ленюк. Киев: Институт математики НАН Украины, 1997. Вып. 16. С. 54 (на укр. яз.).

[19] V. D. Gordevsky. Math. Meth. Appl. Sci. 1998. V. 21. P. 1479.

Поступила в редакцию 17.IV.2000 г. 